\title{
Implementation and Performance of the High-Level Trigger electron and photon selection for the ATLAS experiment at the LHC
}

\author{
F. Monticelli on behalf of the ATLAS e/gamma group * \\ UNLP, Universidad Nacional de La Plata, \\ La Plata, Argentina \\ E-mail: Fernando.Monticelli@cern.ch
}

\begin{abstract}
The ATLAS three-tier trigger system faces the challenge to reduce the incoming rate of $40 \mathrm{MHz}$ to $\sim 200 \mathrm{~Hz}$. It consists of hardware based Level-1, and a software based High-Level Trigger (HLT). ${ }^{1}$ In this talk an overview of the selection algorithms for electrons and photons will be given as well as the expected performance. The electron and photon trigger menu and the strategy for the initial phase of LHC exploitation.
\end{abstract}

Keywords: High Level Trigger; ATLAS; electron; photon.

\section{Introduction}

The ATLAS experiment will start taking data in 2008 at the Large Hadron Collider (LHC), currently under construction at CERN.

ATLAS $^{2}$ is a multipurpose detector, designed to explore the fundamental nature of matter and to search for new particles such as the Higgs boson and possible unforeseen new physics processes.

The ATLAS detector is equipped with the inner detector (Pixel, SCT silicon strips and TRT straw tubes), the Liquid Argon (LAr) electromagnetic calorimeter, the Tile hadronic calorimeter and the outer muon system. In total there are more than 100 million readout channels.

\footnotetext{
*Anduaga X., Baines J., Benslama K., Berry T., Coura Torres R., Damazio D., De Santo A., Dova M., Emeliyanov D., Enoque Ferreira de Lima D., Flacher H., Fonseca-Martin T., Gaumer O., Goncalo R., Grabowska-Bold I., Hryn'ova T., Khoriauli G., Kilvington G., Konstantinidis N., Ledroit F., Losada M., Monticelli F., Moreno D., Padilla C., Parodi F., Perez Reale V., Pinzon G., Riu I., Rodriguez D., Rogriquez Y., Santamarina C., Schiavi C., Seixas J. M., Sivoklokov S., Sutton M., Tripiana M., Wang M., Watson A., Wielers M., Woehrling E.-E., Wu X., Ozcan E.,
} 
The high number of detector channels, leads to a mean event size of $\sim$ 1.5 MB. The trigger and data acquisition system (TDAQ) will reduce the incoming rate to $\sim 200 \mathrm{~Hz}$ which is the rate allocated for offline processing.

\section{The ATLAS High Level Trigger}

In the ATLAS experiment, the reduction of the $40 \mathrm{MHz}$ incoming rate down to the $200 \mathrm{~Hz}$ maximum event data storage rate will be provided through three different trigger levels.

The hardware based first level trigger (LVL1) performs a preliminary rejection using only reduced granularity data coming from the calorimeters and the muon detector. It operates within a latency $\sim 2.5 \mu \mathrm{s}$, producing an average output rate of $75 \mathrm{kHz}$ upper limited to $100 \mathrm{kHz}$. Further event selection is done by the software based second level trigger (LVL2) and event filter (EF), collectively referenced as the HLT. The reconstruction at LVL2 is seeded by the LVL1 result using full granularity of the ATLAS subdetectors, processing data contained in the regions of the detector identified by the LVL1 as regions of interest (RoIs), each correspond to $\sim 2 \%$ of the detector. The LVL2 is designed to have an output rate less than $2 \mathrm{kHz}$, with a mean processing time of $40 \mathrm{~ms}$. The EF, seeded by the LVL2 results, uses more complex reconstruction.

The main two concepts characterizing the event selection at the HLT in ATLAS are the RoI guided and step wise reconstruction. This event selection mechanism reduces the amount of data to be processed at each trigger stage. Another feature of the ATLAS HLT event selection strategy is the early rejection achieved by alternating reconstruction algorithms and the so-called hypothesis algorithms which decide if the candidate fulfills certain identification criteria. The event can be rejected after any hypothesis step.

\section{Electron and photon selection}

Electron and photon reconstruction mainly exploits data coming from the electromagnetic calorimeter and the Inner Detector (ID) tracking systems. The starting point for the electron and photon chains are the triggered LVL1 electromagnetic cluster candidate. The first step in the $e / \gamma$ chain is a fast clustering algorithm which is seeded by the LVL1 RoIs.In the next step a hypothesis algorithm uses this information to perform preliminary particle identification. In case the event is accepted, the $e / \gamma$ chain then continues with the EF processing. For electrons track reconstruction is performed in 
the ID. There are two main tracking algorithms being studied: IDScan and SiTrack ${ }^{4}$ which have very similar performances. In the next step, cluster to track association is done using the matching between the cluster positions with the corresponding $\eta$ and $\phi$ values of the tracks extrapolated to the calorimeter surface. In the subsequent step a particle identification is done based on the $p_{T}$ of the track, the $E_{T} / p_{T}$ ratio and the position match between the calorimeter cluster and the extrapolated track. The EF re-uses the available offline algorithms with more precise calibrations and alignment constants than at LVL2. Electron and photon trigger menus have been set to be fully efficient in all the pseudorapidity region $0<|\eta|<2.5$.

\section{Performance of electron and photon trigger}

Electron and photon trigger menus have been extensively studied for luminosity $\mathrm{L} \approx 10^{33} \mathrm{~cm}^{-2} \mathrm{~s}^{-1}$. It was demonstrated they are well set-up to trigger on events produced at HLT. Table 1 shows the trigger efficiency w.r.t. to the offline selection for a low mass Higgs decaying into two photons selected by either the trigger which efficiently selects photons above a transverse energy of $\mathrm{ET}=60 \mathrm{GeV}$ (g60) or by the trigger which requests two isolated photons selected efficiently with ET $>20 \mathrm{GeV}(2 \mathrm{~g} 20 \mathrm{i})$. In the same table the respective expected background rate is also shown. Table 2 shows the expected electron trigger efficiencies for $\mathrm{Z} \rightarrow e^{+} e^{-}$and for Exotic Gravitons $\mathrm{G} \rightarrow e e$ of $500 \mathrm{GeV}$ invariant mass.

Table 1. Photon trigger efficiencies w.r.t. to the offline photon selection for $\mathrm{H}_{120} \rightarrow \gamma \gamma$

\begin{tabular}{|c||c|c||c|c|}
\hline \multicolumn{1}{|c||}{ Trigger } & \multicolumn{2}{c||}{$2 \mathrm{~g} 20 \mathrm{i}$} & \multicolumn{2}{c|}{ g60 } \\
Level & Eff & Rate & Eff & Rate \\
\hline \hline L1 & $96.4 \pm 0.5 \%$ & $150 \pm 10 \mathrm{~Hz}$ & $92.9 \pm 0.4 \%$ & $1200 \pm 80 \mathrm{~Hz}$ \\
\hline L2 & $94.6 \pm 0.7 \%$ & $5 \pm 1.7 \mathrm{~Hz}$ & $86.8 \pm 0.6 \%$ & $35 \pm 14 \mathrm{~Hz}$ \\
\hline EF & $93.8 \pm 0.8 \%$ & $2 \pm 1 \mathrm{~Hz}$ & $84.7 \pm 0.6 \%$ & $16 \pm 9 \mathrm{~Hz}$ \\
\hline
\end{tabular}

In March and May 2007 the timing of the LVL2 and EF algorithms were measured in a so-called technical run. During this period the trigger software was run in playback mode on simulated events using a subset of the final HLT farm. Figure 2 show the LVL2 and EF total time measured for accepted events. The LVL2 timing obtained in the technical run in May 2007 was around $100 \mathrm{~ms}$ with a large RMS. The EF mean processing time was $1.57 \mathrm{~s}$. Work is ongoing to improve further the timing performance of the HLT algorithms. 
Table 2. Electron trigger efficiencies w.r.t. offline electron selection for $\mathrm{Z} \rightarrow e^{+} e^{-}$and $\mathrm{G} \rightarrow e e$

\begin{tabular}{|c||c||c|c|}
\hline \multicolumn{1}{|c||}{ Sample } & \multicolumn{2}{c||}{$\rightarrow e^{+} e^{-}$} & \multicolumn{2}{c|}{$\mathrm{G} \rightarrow e e$} \\
\cline { 1 - 1 } Trigger Level & $\mathrm{e} 25 \mathrm{i}$ & $2 \mathrm{e} 15$ & $\mathrm{e} 60$ \\
\hline \hline $\mathrm{L} 1$ & $96.0 \%$ & $99.9 \pm 0.1$ & $99.9 \pm 0.1$ \\
\hline $\mathrm{L} 2$ & $84.4 \%$ & $84.4 \pm 0.5$ & $96.1 \pm 0.2$ \\
\hline $\mathrm{EF}$ & $84.0 \%$ & $73.0 \pm 0.6$ & $92.1 \pm 0.3$ \\
\hline
\end{tabular}

Note: Using IDScan algorithm for tracking

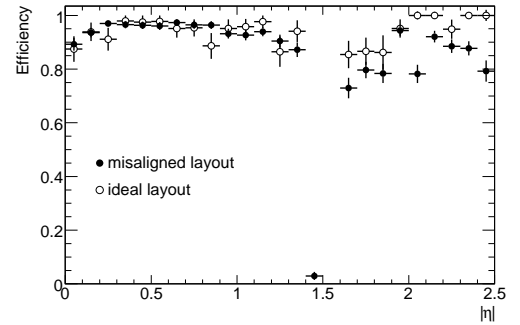

(a)

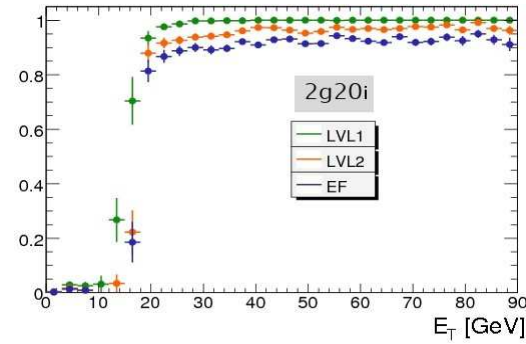

(b)

Fig. 1. (a) e25i trigger efficiency vs pseudorapidity $|\eta|$ w.r.t. offline; (b) g20i trigger efficiency vs $p_{T}$ after each trigger level w.r.t. offline

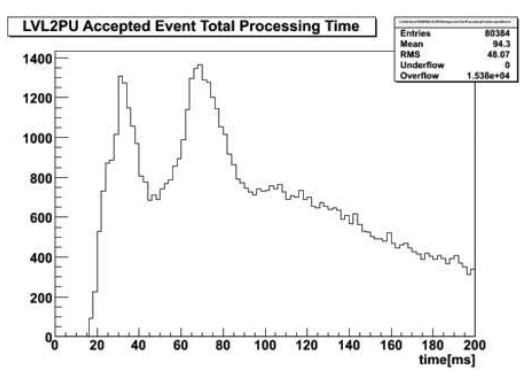

(a)

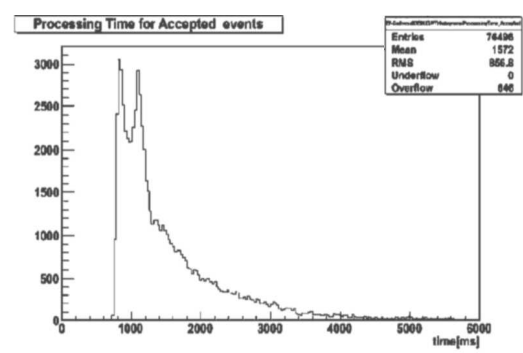

(b)

Fig. 2. Total time for accepted events measured for (a) LVL2 and (b) EF

\section{Strategy at start up}

At start-up an initial luminosity of $\mathrm{L} \approx 10^{31} \mathrm{~cm}^{-2} \mathrm{~s}^{-1}$ is expected. The startup trigger menu for electrons and photons has to provide the data samples needed to commission the trigger and detectors and provide useful data to be used for physics analysis. Therefore, a good selection of the physics 
channels $J / \Psi \rightarrow e e, Y \rightarrow e e$, Drell-Yan, $Z \rightarrow e e, W \rightarrow e \nu$, and direct photon production will be crucial. The aim is to select events with at least one electron above $\approx 10 \mathrm{GeV}$ or one photon above $\approx 20 \mathrm{GeV}$ in addition to the relevant double-object triggers, e.g. for selecting $J / \Psi, Y$, and $\mathrm{Z}$ events. As the data as well as the trigger and detector performance need to be understood the trigger selections use loose selection criteria. The photon triggers will use the same loose cuts for the calorimeter based selections. A range of signatures are foreseen in the trigger menu to adapt to running conditions. If the rate is too high triggers with higher thresholds are defined and/or pre-scales can be adjusted. To ensure the selection of important physics channels, redundant triggers are present, for example, a dedicated $J / \Psi$ trigger. For $W \rightarrow e \nu$, triggers which combine electrons and missing transverse energy are available. With time the trigger and detector performance will be understood and gradually the trigger selections can be tightened and some redundancy triggers can be dropped.

\section{Conclusions}

The ATLAS trigger performance of electrons and photons at low luminosity $\left(\mathrm{L}=10^{33} \mathrm{~cm}^{-2} \mathrm{~s}^{-1}\right)$ has been discussed. It has been shown the triggers are well set-up for the various physics processes with electrons and photons in the final state. The HLT algorithms have been tested using simulated events in a subset of the final HLT farm. This has been an important test for the timing performance and a final test to ensure the algorithms are set-up for being run in the real HLT online environment.

\section{References}

1. ATLAS HLT/DAQ/DCS Group, ATLAS High-Level Trigger, Data Acquisition and Controls Technical Design Report, CERN/LHCC/2003-022, ATLASTDR-016 (2003).

2. ATLAS Collaboration, ATLAS Detector and Physics Performance Technical Design Report, CERN/LHCC/99-14, ATLAS-TDR-014 (1999).

3. G. Comune et al, The Algorithm Steering and Trigger Decision mechanism of the ATLAS High Level Trigger, ATLAS Internal Note, ATL-DAQ-2003-031, Proceedings CHEP03, La Jolla, California, March 24-28, 2003.

4. N. Konstantinidis et al, Fast Tracking for the ATLAS LVL2 Trigger, ATLDAQ-CONF-2005-001, proceedings of the Computing in High Energy Physics and Nuclear Physics 2004 Conference, Interlaken, Switzerland, 27 September - 01 October 2004; C. Schiavi et al 